\title{
Hydrogen sulfide inhibits myocardial injury induced by homocysteine in rats
}

\author{
Lin Chang $\cdot$ Bin Geng $\cdot$ Fang Yu $\cdot$ Jing Zhao $\cdot$ \\ Hongfeng Jiang $\cdot$ Junbao Du $\cdot$ Chaoshu Tang
}

Published online: 29 January 2008

(C) Springer-Verlag 2008

\section{Erratum to: Amino Acids \\ DOI 10.1007/s00726-007-0011-8}

The present Fig. $6 b$ shows that $\mathrm{H}_{2} \mathrm{~S}$ was added at early time points (i.e.100 time/S). This was an oversight. Hcy induced a transient superoxide anion release in isolated myocardial mitochondria. However, in the early times, we found that $\mathrm{H}_{2} \mathrm{~S}$ could not be added and a curve was not observed. So, we pre-incubated mitochondria with different concentrations of $\mathrm{H}_{2} \mathrm{~S}$, followed by the addition of Hcy. We found that pre-treatment with $\mathrm{H}_{2} \mathrm{~S}$ at $10^{-9} \mathrm{~mol} / \mathrm{L}$, mostly blocked superoxide anion release induced by Hcy, and treated with $\mathrm{H}_{2} \mathrm{~S}$ from $10^{-8}$ to $10^{-4} \mathrm{~mol} / \mathrm{L}$ completely abolished superoxide anion production, that is, the tracings were not elevated and remained at baseline.

The correct Fig. 6 is given below:

The online version of the original article can be found under doi:10.1007/s00726-007-0011-8.

\section{Chang $\cdot$ B. Geng $(\bowtie) \cdot$ C. Tang}

Institute of Cardiovascular Research, Peking University,

Xishuku Street 8, West District, 100034 Beijing,

People's Republic of China

e-mail: bingeng@bjmu.edu.cn

B. Geng $\cdot$ F. Yu $\cdot$ J. Zhao $\cdot$ H. Jiang $\cdot$ C. Tang

Department of Physiology,

Peking University Health Science Center,

Beijing, People's Republic of China

J. Du

Department of Pediatric, First Hospital of Peking University,

Beijing, People's Republic of China
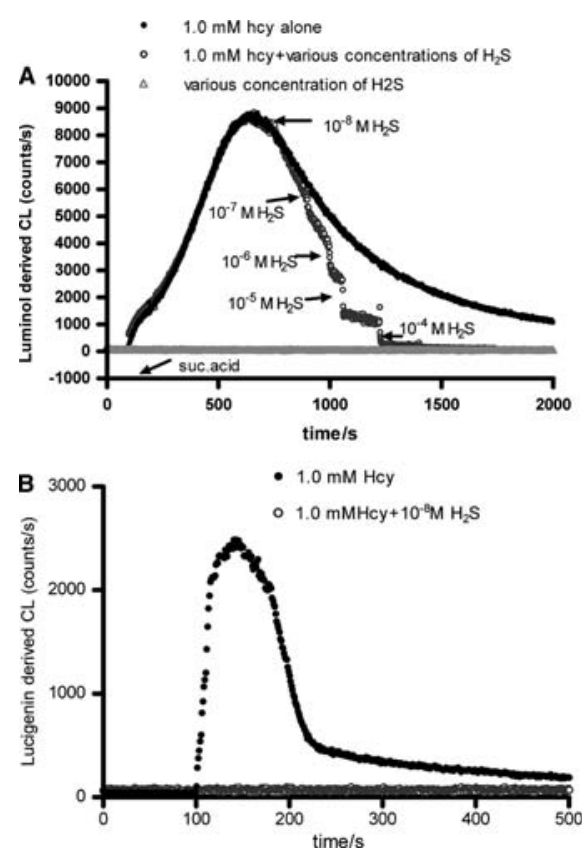

Fig. 6 Hydrogen sulfide cleaved reactive oxygen species produced by Hcy in myocardial mitochondria. a Myocardial mitochondria were isolated from normal rat hearts and incubated with $0.1 \mathrm{mmol} / \mathrm{L} \mathrm{Hcy}$ for 5 min followed by $\mathrm{H}_{2} \mathrm{O}_{2}$ production triggered by succinate acid. At peak $\mathrm{H}_{2} \mathrm{O}_{2}$ production, various dosages of $\mathrm{H}_{2} \mathrm{~S}$ were added in the incubation buffer, step by step, from low to high dosage. The $\mathrm{H}_{2} \mathrm{O}_{2}$ production curve was monitored by computerized chemilal machine. b Isolated myocardial mitochondria were incubated with $0.1 \mathrm{mmol} / \mathrm{L}$ Hcy for 5 min followed by measuring the superoxide anion production by lucigenin-derived chemiluminescence. At the peak of the curve, different concentrations of $\mathrm{H}_{2} \mathrm{~S}$ were added and the alteration in superoxide anion was recorded. An amount of $0.1 \mathrm{mM}$ hypoxanthine plus $10 \mathrm{mU}$ xanthine oxidase induced superoxide anion was used as a positive control 